\title{
Proinflammatory Cytokine Expression Contributes to Brain Injury Provoked By Chronic Monocyte Activation
}

\author{
Anna-Leena Sirén, ${ }^{1,2,3}$ Richard McCarron, ${ }^{2,4}$ Lan Wang, ${ }^{1}$ Patricia Garcia-Pinto, ${ }^{1}$ Christl Ruetzler, ${ }^{2}$ \\ David Martin, ${ }^{5}$ and John M. Hallenbeck ${ }^{2}$ \\ ${ }^{1}$ Department of Neurology, The Uniformed Services University of the Health Sciences, 4301 Jones Bridge \\ Road, Bethesda, MD 20814, USA \\ ${ }^{2}$ Stroke Branch, National Institute of Neurological Disorders and Stroke, NIH, 9000 Rockville Pike, \\ Bethesda, MD 20892, USA \\ ${ }^{3}$ Departments of Psychiatry and Neurology, University of Goettingen and the Max-Planck-Instifute for \\ Experimental Medicine, Hermann-Rein-Str. 3, D-37075-Goettingen, Germany \\ ${ }^{4}$ Naval Medical Institute, 8901 Wisconsin Ave, Bethesda, MD 20889, USA \\ ${ }^{5}$ Department of Pharmacology, MS-15-2-A., Amgen Inc., One Amgen Center Drive, Thousand Oaks, \\ CA 91320, USA
}

Accepted December 20, 2000.

\begin{abstract}
Background: We have proposed that an increased interaction between monocyte/macrophages and blood vessel endothelium predisposes subjects to strokes. The effect of chronic monocyte activation on the development of cerebral infarcts was thus studied in rats after provocation of a modified local Swartzman reaction, in brain vasculature. Materials and Methods: Two weeks after an IV bolus of bacillus Calmette-Guérin (BCG), we studied spontaneous superoxide production, integrin expression, endothelial adhesion of monocytes and the neurological symptoms, brain histology, and cytokine immunoreactivity after a provocative dose of LPS (30-300 $\mu \mathrm{g} / \mathrm{rat}$ i.c.v.).

Results: Monocyte migration into the brain was stimulated by BCG priming. The incidence of paralysis and death in response to LPS was markedly increased in BCGprimed rats. Histological evaluation of the brains of neurologically impaired and moribund animals revealed
\end{abstract}

intravascular thrombosis and pale and hemorrhagic infarcts. Infiltrates of leukocytes expressing immunoreactive IL-1 $\beta$, IL-6, and TNF- $\alpha$ were found around blood vessels, cerebral ventricles, and meninges, and were accompanied by a profound microglial expression of IL $1 \beta$, endothelial expression of IL-6, and expression of TNF- $\alpha$ and TNF-R 1 in glia and neurons of cortex and hippocampus. Treatment $(2 \times 100 \mu \mathrm{g} / 10 \mu$ l, i.c.v. $)$ with recombinant human (rh-)TNF $55 \mathrm{kDa}$ receptor completely prevented, and treatment with rh-IL-1 receptor antagonist significantly decreased the incidence of paralysis and death in response to BCG + LPS. The improvement of neurological symptoms was accompanied by reduced histological damage and supppression of IL- $1 \beta$ expression in the brain tissue.

Conclusions: The data demonstrate that chronic monocyte activation predisposes subjects to thrombosis and hemorrhage via an exaggerated release of proinflammatory cytokines.

\section{Introduction}

We have proposed that stroke-risk factors may increase the interactions between circulating monocytes and blood vessel endothelium via cytokines and that this can predispose local segments of extracranial and intracranial vessels to subsequent thrombosis or hemorrhage $(1,2)$. Several established risk factors for stroke (hypertension, old age, diabetes, genetic stroke-proneness) increased the incidence of ischemia and hemorrhage in brainstem vasculature of rats in response to a "provocative" dose of lipopolysaccharide (LPS) (1). Furthermore,

Address correspondence and reprints to: Dr. Sirén, Max-PlanckInstitute for Experimental Medicine, Hermann-Rein-Str. 3, 37075 Goettingen, Germany. Phone: 011-49-551-3899627.

Fax: 011-49-551-3899670. E-mail: siren@em.mpg.de monocyte activation and an increased adherence of monocytes to endothelium are induced by risk factors such as hypertension (3-7). Accordingly, an increased local accumulation of monocyte/ macrophages has been found in the brains of aged or stroke-prone and hypertensive rats (8); cells of these rats produced more TNF- $\alpha$ in response to stimulation (with LPS) than cells from normotensive control animals $(2,6,9)$.

The present study addresses specifically the pathophysiological role of chronic monocyte/ macrophage activation in the development of local thrombosis and hemorrhage using the modified Shwartzman paradigm in rats $(1,10,11)$. Chronic monocyte activation was induced by injection of Bacillus Calmette Guerin (BCG) organisms, which are known to cause a prolonged activation of 
macrophages in normal rodents (12-14). We hypothesized that monocyte/macrophage activation could lead to increased adhesion and transendothelial migration of monocytes and an exaggerated release of IL1 and TNF- $\alpha$ from monocyte/macrophages located around local blood vessels. This would mediate preparation of those vessels for the focal thrombosis and/or hemorrhage, as occurs in the local Shwartzman reaction. Another aspect of these experiments was to test whether inhibition of IL-1 or TNF- $\alpha$ activity could reduce the pathological changes and stroke incidence in this model.

\section{Material and Methods}

Male Sprague-Dawley rats (225-250 g) were purchased from Taconic Farms (Germantown, NY). The animals were housed at $22^{\circ} \mathrm{C}$ with a $12 \mathrm{hr}$ light$12 \mathrm{hr}$ dark cycle with food and tap water ad libitum.

\section{Animal Procedures}

To activate mononuclear cells, an intravenous bolus injection $1 \mathrm{~mL}$ of Bacillus Calmette Guerin (BCG) suspension (Trudeau Institute, Inc., Phipps strain 1029, Sarance Lake, NY, approximately $2.2 \times$ $10^{8}$ organisms $/ \mathrm{mL} / \mathrm{vial}$ ) was administered into the tail vein 2 weeks before LPS administration. This time period between BCG priming and subsequent LPS provocation was chosen since BCG infection in rodents is known to be maximal 2-3 weeks after BCG inoculation (15-18). This was further verified by our studies that demonstrated increased sensitivity to LPS-induced paralysis 2 weeks but not 1 or 4 weeks after BCG priming (see Results). The control rats received the same volume of sterile saline injection into the tail vein. The monocyte activation was monitored by nitroblue tetrazolium reduction test (NBT), a measure of spontaneous superoxide production (3), by expression of activation markers (CDIlb, CDllc, CD18) using flow cytometry (5), and by monitoring monocyte adhesion to cultured cerebrovascular endothelial cells (5).

Rats were anesthetized with halothane $(4 \%$ in $100 \%$ oxygen). For intravenous injections, a sterile PE-50 catheter was inserted into the femoral vein under aseptic conditions. Lipopolysaccharide E. coli Olll:B4 (phenol extracted, Sigma, St. Louis, MO) dissolved in $0.9 \% \mathrm{NaCl}$ (sterile, pyrogen-free) was injected into the lateral cerebral ventricles. For this procedure, the rat was placed in a stereotaxic device (David Kopf Instruments, Tujunga, CA). A midline cut was then made on the scalp to expose the parieto-occipital bone area and the muscles attached to the occiput were gently separated to expose the atlanto-occipital membrane. A stainless steel guide cannula was placed on the right parietal skull (coordinates from bregma: anteroposterior $=0.8 \mathrm{~mm}$, lateral $=1.2 \mathrm{~mm}$ ) and fixed with glue. Study Protocol: Lipopolysaccharide (dissolved in sterile, pyrogen-free $0.9 \% \mathrm{NaCl}$ ) was injected in a total volume of $10 \mu \mathrm{l}$ by means of a premeasured $30 \mathrm{~g}$ cannula $(7.5 \mathrm{~mm})$ over a period of $5 \mathrm{~min}$. Two additional minutes were allowed for dispersal of the injected fluid before withdrawal of the cannula. The two anticytokine treatments, polyethylene glycolconjugated dimer of recombinant soluble human TNF 55kDa receptor (PEG-[rsTNF-RI] ${ }_{2}$ ) designated as TNF binding protein (TNFbp) and the recombinant human IL-1 receptor antagonist (IL-lra) were kindly provided by Amgen, Inc. (Boulder, CO). The stock solution of TNFbp $(10 \mathrm{mg} / \mathrm{mL})$ was provided in saline and used undiluted. Sterile phosphate buffered saline (PBS), pH 7.4, was used as the vehicle control for TNFbp. The stock solution of ILl-ra $(100 \mathrm{mg} / \mathrm{mL})$ was provided in an acidic buffer solution $(10 \mathrm{mM}$ sodium citrate, $140 \mathrm{mM}$ sodium chloride and $0.5 \mathrm{mM}$ EDTA, $\mathrm{pH} 6.5$ ) and was diluted 1:10 with sterile PBS. Sterile PBS, pH 6.5, was used as the vehicle control for IL-1ra. IL-1ra $(100 \mu \mathrm{g} / 10 \mu \mathrm{l})$ and TNFbp $(100 \mu \mathrm{g} / 10 \mu \mathrm{l})$, or corresponding vehicle solutions were administered i.c.v. in a volume of $10 \mu \mathrm{l}$ $30 \mathrm{~min}$ before and $30 \mathrm{~min}$ after a single bolus i.c.v. injection of LPS (100 $\mu \mathrm{g} / \mathrm{rat})$.

Prior to the i.c.v. injections, all rats underwent a baseline neurological examination previously described in detail (Hallenbeck et al., 1988). This scoring of function is derived from the method of Tupper and Wallace (1980). Based on observed deficits, a functional grade is assessed: Grade 0, normal rat; Grade 1, lethargy, no signs of paresis, spontaneous movement greatly reduced; Grade 2, clear signs of paresis in at least one limb but able to walk; Grade 3, severe paresis/paralysis, unable to walk; Grade 4, dead. The rats were examined again at $2 \mathrm{hr}, 4 \mathrm{hr}$, $6 \mathrm{hr}, 8 \mathrm{hr}$, and $24 \mathrm{hr}$ after the i.c.v. injection of LPS.

\section{Monitoring of Monocyte Activation}

For measurement of spontaneous monocyte superoxide production, a $100 \mu \mathrm{l}$ volume of the venous blood was drawn from the tail vein before the BGC injection and 2 weeks later. The blood was mixed with an equal amount of $0.2 \%$ sodium nitroblue tetrazolium solution and incubated at $37^{\circ} \mathrm{C}$ for $30 \mathrm{~min}$ followed by a incubation for $15 \mathrm{~min}$ at room temperature. After gentle stirring of the solution, cover slip smears were made and stained with Wright's stain, and 30 monocytes were counted at x100 magnification.

For flow cytometry and adhesion assays, peripheral blood was obtained by puncture of the abdominal aorta and collected into heparin-treated vials. Mononuclear cells were isolated by FicollHypaque (Histopaque 1119/Histopaque 1077, Sigma) density gradient centrifugation and further purified by centrifugation on hyperosmotic Nycodenz (Accurate Chemical and Scientific, Westbury, NY) density gradients as previously described (5). Monocytes were washed with PBS containing $0.5 \%$ bovine serum albumin and $2 \%$ EDTA to remove 
platelets. The monocytes were counted, and their purity was assessed by FACS analysis using an antibody to rat monocytes (ED-1, Chemicon, Temecula, CA). Analysis of cell surface molecule expression on freshly prepared monocytes was performed by staining with rhodamine-labeled antibodies and analyzed by flow cytometry using a FACS IV analyzer (Becton-Dickinson FACS System, Mountain View, CA). The data are presented as mean fluorescence intensity (MFI) representing semiquantitative data (in channel units) on the mean number of fluorescent reagent-binding molecules expressed by the cells.

Monocytes used in adhesion experiments were labeled with sodium ${ }^{51} \mathrm{Cr}$-chromate $(0.1 \mathrm{mCi} / \mathrm{mL})$ for $30 \mathrm{~min}$ at $37^{\circ} \mathrm{C}$, washed twice with warm Hank's balanced salt solution (HBSS) containing $4 \%$ fetal calf serum (FCS), and added $\left(2 \times 10^{4} / 50 \mathrm{~mL} / \mathrm{well}\right)$ to monolayers of rat cerebrovascular endothelial cells in microtiter plates, which were isolated and cultured as described previously (5). Endothelial cells were cultured at $37^{\circ} \mathrm{C}$ in media alone or with media containing human recombinant TNF- $\alpha$ $(100 \mathrm{U} / \mathrm{mL})$ and IL- $1 \beta(15 \mathrm{U} / \mathrm{mL})$ for $24 \mathrm{hr}$ before addition of ${ }^{51} \mathrm{Cr}$-chromate-labeled monocytes and subsequently incubated at $37^{\circ} \mathrm{C}$ for $30 \mathrm{~min}$. After incubation, nonadherent cells were removed, endothelial cell monolayers were washed, and adherent cells were lysed with $2 \%$ (vol/vol) Triton-X $(0.2 \mathrm{~mL} /$ well $)$. Radioactivity in all fractions was counted on a LKB Compugamma 1282 gamma counter. The number of adherent cells was evaluated by the measured ratio of counts/min to cell counts established for each experiment using known numbers of monocytes. These numbers were verified with calculations for percentage of adherent cells calculated by dividing Triton-X counts/min by the total counts/min added. All experiments were performed in triplicate. More than $90 \%$ of the cells adhering to endothelial cell monolayers stained positively for the rat monocyte marker ED-1, indicating that adhesion by contaminating lymphocytes was minimal. In some experiments, endothelial cell monolayers were preincubated $\left(30 \mathrm{~min}\right.$ at $\left.37^{\circ} \mathrm{C}\right)$ with a saturating concentration $(25 \mathrm{mg} / \mathrm{mL})$ of the monoclonal antirat ICAM-1 antibody (1A29, Seikagaku America, Rockville, MD) and washed three times with warm HBSS containing $4 \%$ fetal calf serum.

\section{Tissue Preparation}

Rats were sacrificed by an i.p. injection of pentobarbital sodium, and the upper torso was perfused through the heart with ice-cold PBS for 10 min followed by perfusion with $4 \%$ paraformaldehyde in PBS for $15 \mathrm{~min}$. The brains were removed and postfixed in $4 \%$ paraformaldehyde PBS overnight, cut into $5-\mathrm{mm}$ blocks, and processed for paraffin embedding. Seven $\mu \mathrm{m}$ thick sections were cut on a microtome. For histological assessment of tissue dam- age, the sections were stained with Cresyl violet or hematoxylin-eosin.

\section{Immunohistochemical Staining}

The sections were heated in $60^{\circ} \mathrm{C}$ for $30 \mathrm{~min}$ and deparaffinized in $100 \%$ xylene at $60^{\circ} \mathrm{C}$ for $30 \mathrm{~min}$. They were further washed in fresh xylene at room temperature $2 \times 5 \mathrm{~min}$. After rehydration through graded ethanols, the endogenous peroxidase activity was blocked in $3 \% \mathrm{H}_{2} \mathrm{O}_{2}$ for $10 \mathrm{~min}$, and the sections were washed with distilled water and phosphate buffered saline (PBS) and treated with $0.1 \%$ trypsin (Sigma) in PBS for $10 \mathrm{~min}$. After rinses in distilled water and PBS, the sections were incubated with $10 \%$ blocking serum in a humid chamber for $\mathrm{l} \mathrm{hr}$ at room temperature. The antibodies were diluted with PBS containing $2 \%$ blocking sera. Sections were incubated with the primary antibody $24 \mathrm{hr}$ at $4^{\circ} \mathrm{C}$. They were then rinsed and washed twice for 10 min with PBS. Amplification of the signal was carried out by the avidin-biotin (ABC) method with the appropriate peroxidase kit (Vector Laboratories, Inc., Burlingame, CA) and visualized with diaminobenzidine (DAB; Sigma) and $0.003 \%$ $\mathrm{H}_{2} \mathrm{O}_{2}$ in $0.1 \mathrm{M}$ TrisHCl (pH 7.6). After rinsing or being rinsed with PBS, the slides were dehydrated through graded alcohols and cleared with xylenes, mounted with Eukitt (O. Kindler GmbH \& Co., Freiburg, Germany), and coverslipped for investigation under a light microscope.

The following polyclonal antibodies in a dilution of 1:500 were used: Polyclonal goat anti-rat IL- $1 \beta$ (R\&D Systems Inc., Minneapolis, MN,), polyclonal goat anti-rat TNF- $\alpha$ (R\&D Systems), polyclonal rabbit anti-rat IL-6 (Endogen, Cambridge, MA), polyclonal goat anti-rodent TNF-RI (E20) (Santa Cruz Biotechnology, Inc., Santa Cruz, CA). The following monoclonal antibodies were used: mouse anti-rat ED-1 (Chemicon) and mouse in a dilution of 1:100, and mouse anti-rat ICAM-1 (RED Systems) in a dilution of 1:500. The biotinylated second antibodies were appropriate $\mathrm{F}\left(\mathrm{ab}^{\prime}\right)_{2}$ fragment specific immunoglobulins (Vector).

The controls for immunohistochemistry included the following: 1) omission of the first antibody, 2) incubation of adjacent sections with immunoglobulins of the same immunoglobulin (sub)class as the primary antibodies in a corresponding dilution, and 3 ) preadsorption of the primary antibodies overnight with 20- to 50-fold excess of the corresponding recombinant rat cytokine (RED Systems Inc.) or with a 100-fold excess of the appropriate control peptide (Santa Cruz Biotechnology Inc.).

The number of IL-1 $\beta$-immunoreactive cells was assessed quantitatively using a computerized densitometry analysis system (NIH Image 1.6) linked to a Olympus microscope at a final magnification of 20x using a MTI CCD72 camera and a Data Translation QuickCapture frame grabber card in a Macintosh 7100/66 computer (Apple Computer, Cupertino, CA). 
Four cortical or two hippocampal fields from two brain sections at the level of the hippocampus were averaged and expressed as counts per $\mathrm{mm}^{2}$ area for each animal (3-5 animals in each group were counted).

\section{Statistical Analysis}

Data in the text are means \pm standard error of the mean (SEM) for the given number of rats. Analysis of variance and Student's two-tailed $t$-test with Bonferoni correction for multiple comparisons were used to compare differences in monocyte activity. Fisher's exact probability test was used to test for differences in clinical outcome between treatment groups.

\section{Results}

\section{Effect of BCG Priming on Monocyte Activity}

Before BCG or saline injections, total monocyte counts were $52 \pm 10$ cells $/ \mathrm{mm}^{3}$. BCG priming induced a significant increase in both total and activated monocyte counts (Figure 1, A and B). The number of granulocytes or lymphocytes was not increased after BCG injection (data not shown). Two weeks after BCG priming, the total monocyte counts were $360 \pm 26$ cells $/ \mathrm{mm}^{3}$, and more than $94 \%$ of these cells were activated as assessed by spontaneous superoxide production of monocytes in whole blood (Figure 1B). Expression of $\beta_{2}$-class integrins on freshly isolated resting monocytes from the BCGtreated rats was 2.0- to 2.3-fold greater than on monocytes from saline-treated rats (Figure 1C). Accordingly, adhesion of BCG-primed monocytes to both unstimulated and cytokine-stimulated brain microvascular endothelial cell cultures was greater than that of monocytes from saline-treated control rats (Figure 1D). The adhesion of both BCG and saline-treated monocytes to cytokine-treated endothelial monolayers was blocked by monoclonal anti-ICAM-1 antibodies, which indicates that the adhesion was mediated by binding of the CDIlb/ CD18 to ICAM-1 on endothelial cells.

\section{Effect of BCG Priming on LPS-induced Paralysis and Death}

The normal SD rats were not vulnerable to paralysis or death after receiving the $30-100 \mu \mathrm{g} / \mathrm{rat}$ doses of LPS, and after receiving the $300 \mu \mathrm{g} / \mathrm{rat}$ dose, only $13 \%$ of the rats were affected (Figure $2 \mathrm{~A}$ ). When identical doses of LPS were injected into the cerebral ventricles of rats that had been primed with BCG 2 weeks before, the rats demonstrated severe neurological deficits, which always resulted in death (clinical grades 3 and 4) in a dose-related manner (Figure 2A). Priming with BCG 1 week $(n=2)$ or 4 weeks $(n=6)$ before a provocative dose of LPS (300 $\mu \mathrm{g} /$ rat, i.c.v.) did not increase LPS-induced neurological deficits. No paralyzed or dead animals were found in these groups after LPS, thus confirming the earlier reports that 2 weeks represent an optimal time lag between BCG priming and LPS challenge

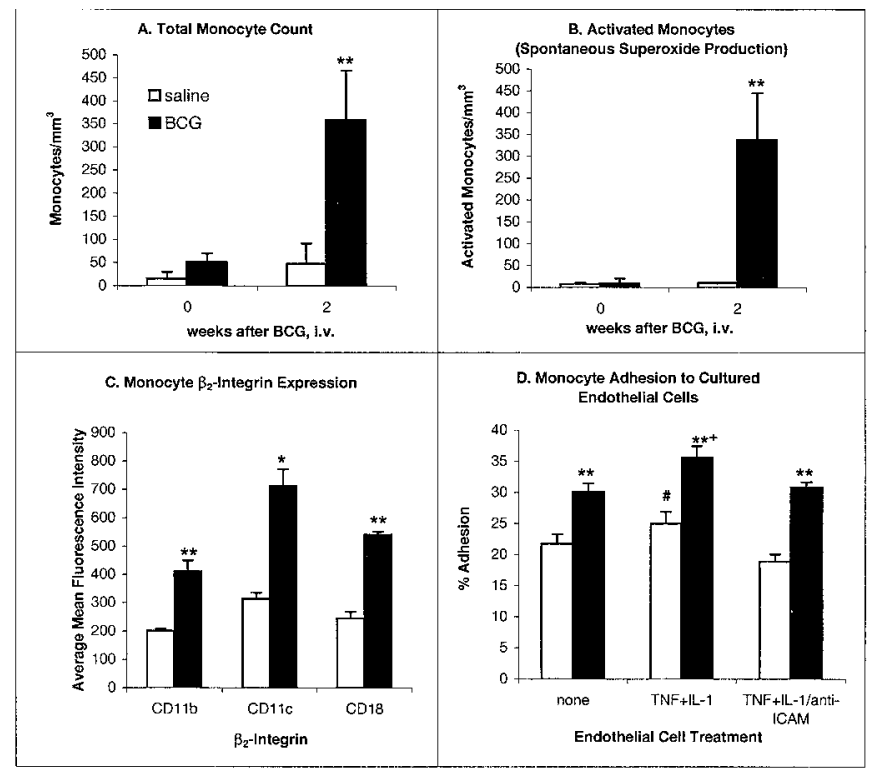

Fig. 1. Monocyte activation in BCG-primed and saline-treated control rats. (A) Total monocyte counts before 2 weeks and after priming. In saline-treated group, $n=4-6$; in BCG-treated group, $n=6-8$. (B) Number of monocytes exhibiting spontaneous superoxide production before and 2 weeks after priming. In saline-treated group, $n=4-6$; in BCG-treated group, $n=6-8$. (C) Flow cytometry analysis of expression of $\beta_{2}$-integrins in freshly isolated monocytes 2 weeks after BCG or saline priming. Data represent means \pm SEM of triplicate samples from two separate experiments. (D) Adhesion of monocytes from BCG or saline-treated rats to cerebromicrovascular endothelial cells. Two weeks after BCG or saline priming, monocytes were isolated and added to untreated, TNF + IL-1 stimulated or to TNF + IL-1 stimulated and anti-ICAMI-treated endothelial cell cultures. Data represent mean \pm SEM of triplicate samples from two separate experiments. Numbers of adherent cells were determined as described in Material and Methods. Asterisks denote statistical significance between saline- and BCG-treated groups; ${ }^{*} p<0.05,{ }^{* *} p<$ 0.01 . In panel $\mathrm{D}$, the cross denotes statistical significance between adhesion of BCG-primed monocytes to TNF + IL1 -treated endothelium and adhesion to untreated or ICAM-1 blocked endothelium; $+p<0.05, p<0.05$ adhesion of saline monocytes to TNF + ILl-treated endothelium $v s$ adhesion to ICAM-1 blocked endothelium. ANOVA followed by Student's $t$-test with Bonferoni correction was used to calculate statistical significance of the changes between the various groups.

(15-18). The time course for deterioration of neurologic function after LPS injections revealed significant impairment in neurologic score starting at $6 \mathrm{hr}$ after LPS administration. Before this time, no animals showed symptoms of paresis or paralysis. They were either normal or slightly lethargic. Most of the BCGprimed animals developed severe symptoms (clinical grade 3 to 4$) 8 \mathrm{hr}$ after $300 \mu \mathrm{g} / \mathrm{rat}$ dose of LPS. However, 4 out of 22 animals were in grade 1 at $8 \mathrm{hr}$ but deteriorated to grades 3-4 at $24 \mathrm{hr}$ after LPS (Figure 2B). Most of the saline-treated rats improved after $6 \mathrm{hr}$ and totally recoved by $24 \mathrm{hr}$ after LPS.

In the animals that received treatments with the TNFbp $(2 \times 100 \mu \mathrm{g} / 10 \mu \mathrm{l})$ prior to and after LPS injection, the clinical deficits and pathological damage 

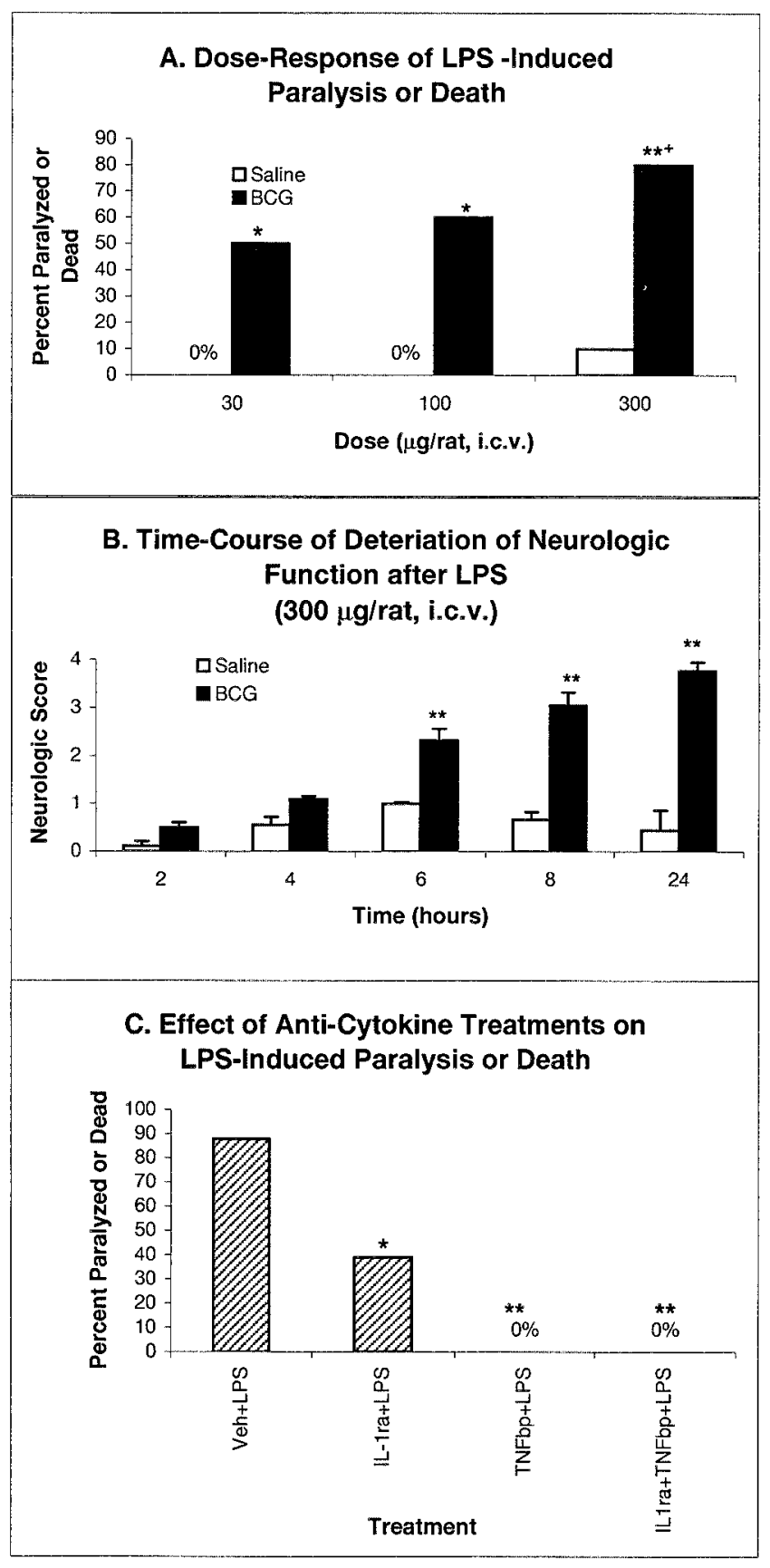

Fig. 2. Neurological function after LPS administration in saline- and BCG-treated rats. (A) Dose-response histograms depicting the incidence of paralysis and death in BCG-treated and control (saline-treated) rats. A single bolus of LPS was administered i.c.v. 2 weeks after the i.v. treatment with BCG or saline. The dose of LPS is indicated on the abscissa. Data represent percent of animals in clinical grades of 3 and 4, $24 \mathrm{hr}$ after LPS administration (all animals that died were paralyzed before their demise). Asterisks indicate statistical significance between the treatment groups, cross denotes difference between LPS 30 and $300 \mu \mathrm{g} / \mathrm{rat}$ dose by 2 -tailed Fisher-exact probability test; $* /+p<0.05,{ }^{* *} p<0.001$. Number of animals was 7-9 in vehicle groups, in BCG groups it was 10, except for the LPS $300 \mu \mathrm{g} / \mathrm{rat}$ dose $(\mathrm{n}=22)$. (B) Cumulative time-course of deteriation of neurologic function after the $300 \mu \mathrm{g} / \mathrm{rat}$ dose of LPS in saline- or BCG-treated rats. Asterisks denote difference between saline- and BCG-treated groups; ${ }^{* *} p<0.01$ (ANOVA were completely abolished. Treatment with IL-1ra $(2 \times 100 \mu \mathrm{g} / 10 \mu \mathrm{l})$ significantly improved the clinical outcome (Figure 2C).

\section{Histopathology of the Brains After LPS Treatment}

Histopathological analysis of brain sections from paralyzed rats revealed infarcts in both gray and white matter along with perivascular cuffing (Figures 3 and 4). Signs of hypoxic-ischemic damage in the cortical areas included vacuolization of the neuropil, with pycnotic, eosinophilic neuronal somas and shrunken hyperchromatic neurons (Figure 3B) along with thrombotic vessels (Figure 3D) and hemorrhages (Figure $3 \mathrm{C}$ ) and perivascular infiltrates of leukocytes (Figure 3A). In one rat, a total occlusion of the right middle cerebral artery (MCA) was accompanied by a pale cortical infarct in the MCA territory (Figure 4, $\mathrm{G}$ and $\mathrm{H}$ ). The meningeal and perivascular cell infiltrates consisted of monocytes because most of these cells expressed the rat monocyte marker EDl (Figure 4, I and N). Patchy expression of ICAM-1 was seen in microvascular endothelium and perivascular glia (Figure 40). Infiltrating inflammatory cells were present in meninges and brain parenchym in rats that showed no neurological deficits or minor reversible deficits (clinical grades 0-1) (Figure 3A). However, no clear neuronal damage or infarcts could be found in the surviving animals' brains (Figure 4, D and E).

Histopathological analysis of the brains of anticytokine-treated rats demonstrated that clinical survival was correlated with absence of profound cellular pathology (Figure 4, J and K). ED 1-positive monocytes and microglia were present around the meninges, cerebral ventricles, and blood vessels (Figure 4L). Except for small intraparenchymal hemorrhages that were occasionally detected in these brains, the parenchymal brain cells were otherwise preserved.

\section{Expression of Cytokine Immunoreactivity in the Brain}

Weak-to-moderate expression of immunoreactivity for IL- $1 \beta$ was present in cortical neurons and hippocampal neuronal fibers of control animals (Figure 5, A-C, Table 1). Immunoreactive IL- $1 \beta$ was also present in

followed by student's $t$-test with Bonferoni correction). Panel C: Effect of TNFbp and IL-1ra on the incidence of LPS-induced paralysis and death in BCG-treated rats. A single bolus injection of LPS (300 $\mu \mathrm{g} / \mathrm{rat}$, i.c.v.) was administered 2 weeks after the BCG priming. The anticytokine treatments, both at a dose of $100 \mu \mathrm{g} / 10 \mu \mathrm{l}$, were administered i.c.v. $30 \mathrm{~min}$ before and $30 \mathrm{~min}$ after the injection of LPS (100 $\mu \mathrm{g} / \mathrm{rat}$, i.c.v.). The various treatment modalities are indicated on the abscissa. Data represent percent of animals in clinical grades of 3 and 4, $24 \mathrm{hr}$ after LPS administration. Asterisks denote statistical significance between anticytokine-treated and vehicle-treated animals by 2 -tailed Fisher exact probability test; ${ }^{*} p<0.05,{ }^{* *} p<0.001$. Number of animals in each drug treatment group was 8; in the vehicle-treated group it was 17 . 

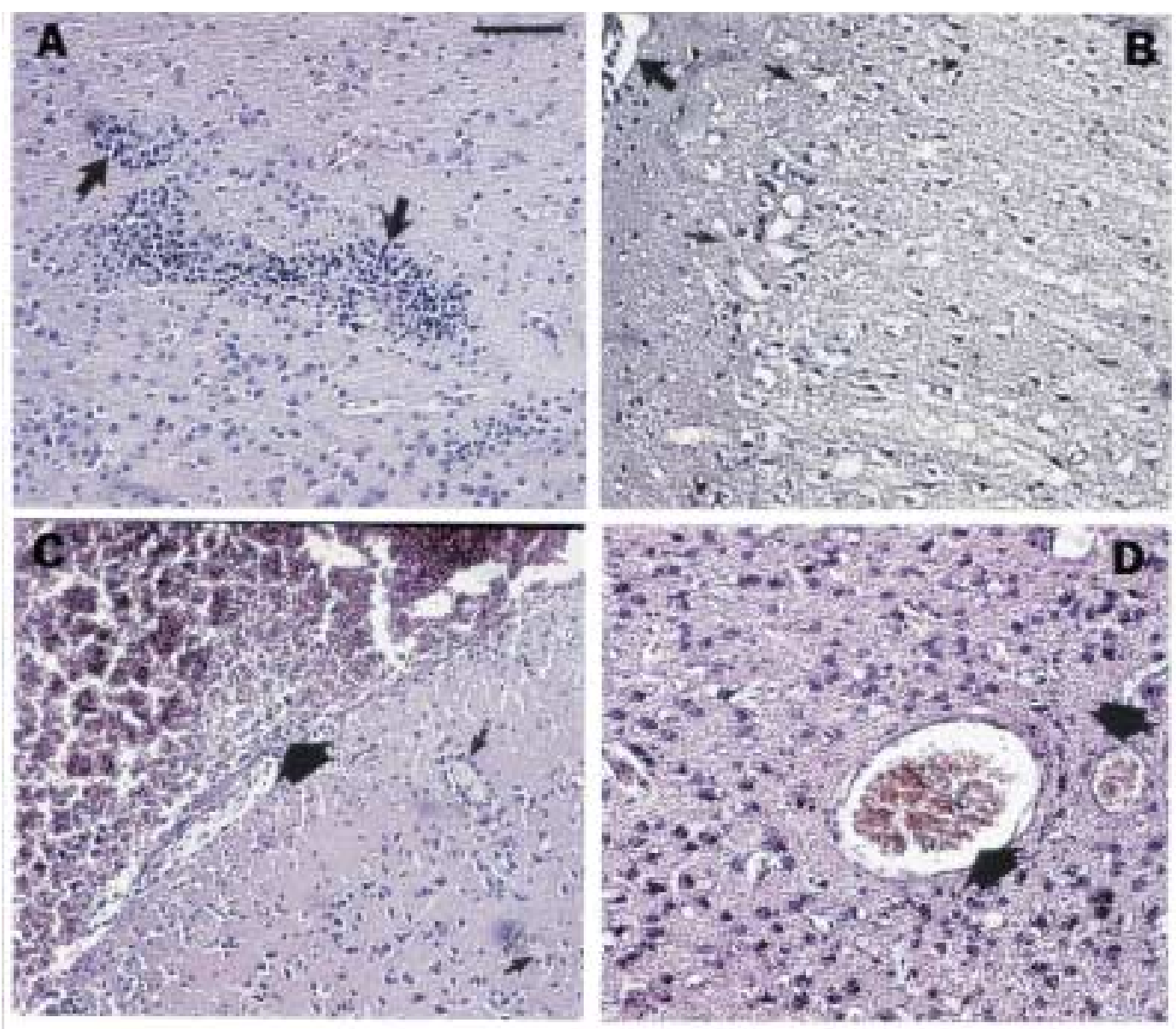

Fig. 3. Photomicrographs demonstrating histological damage after LPS administration ( $300 \mathrm{mg} / \mathrm{rat}$ i.c.v.) in BCG pretreated rats. Perivascular cuffs of inflammatory cells (3A), a cortical infarct (3B, arrow heads), hemorrhage (3C, arrow heads) in subcortical white matter, thrombotic vesssels (3D). Scale bar $=25 \mu \mathrm{m}$, except in C scale bar $=50 \mu \mathrm{m}$.

infiltrating cells around meninges and blood vessels of most LPS/ LPS + BCG-treated animals (Figure 5, $\mathrm{E}$ and $\mathrm{H})$. A strong immunoreactive signal was seen in cells with microglial morphology (Figure 5, E, F, $\mathrm{H}$, and $\mathrm{I}$ ). This staining was restricted to cortex and subcortical white matter in saline + LPS-treated animals (Figure 5D, Table 1). In BCG + LPS-treated rats, strong IL- $1 \beta$ expression was visible thoughout the brain (Figure 5G), in cells with microglial morphology (Figure 5I), as well as in the vascular wall (Figure 5, H, I, and L) and the inflammatory cell infiltrates (Figure 5L). This staining was abolished with preincubation of the antibody with recombinant rat IL-1 $\beta$ (Figure 5J and 5K). Expression of IL$1 \beta$-immunoreactivity was almost totally suppressed in the brains of animals in which anticytokine treatment was effective in preventing paralysis and death after LPS challenge (Figure 5M, N, and O, Table 1)even when staining of adjacent sections with the monocyte-marker EDI revealed presence of monocytes and microglial cells in these brains. (Figure $4 \mathrm{~L}$ and Figure $5 \mathrm{~N}$ represent staining on adjacent sections for $\mathrm{ED} 1$ and $\mathrm{ILl} \beta$, respectively.) In fact, survival of the animals after BCG + LPS treatment was directly correlated with the extent and intensity of microglial IL-1 $\beta$-immunoreactivity.

Weak-to-moderate cytoplasmic immunoreactivity for TNF- $\alpha$ was seen in neurons of both control and LPS- or LPS + BCG-treated rats. The neuronal expression of TNF- $\alpha$-immunoreactivity was not clearly correlated with histopathological changes or clinical grade. The TNF- $\alpha$-immunoreactivity was seen in neurons and round inflammatory cells of the cortex, around blood vessels and meninges, and in the smooth muscle of larger vessels of paralyzed and moribund rats (Figure 6A). This staining was abolished with preincubation of the antibody with recombinant rat TNF- $\alpha$ (Figures 6C), but complexing of the recombinant rat TNF- $\alpha$ with TNFbp prior to preadsoption with the antibody restored the staining (Figure 6D). In the brains of anticytokinetreated animals, TNF- $\alpha$ immunoreactive cells were seen in inflammatory cells and cells with glial morphology (Figure 6B).

Weak-to-moderate TNF-R 1 immunoreactivity was expressed in processes around the meningeal surface, the ventricles and the blood vessels of LPS- and LPS + BCG-treated animals (Figure 6E). The TNFR 1 


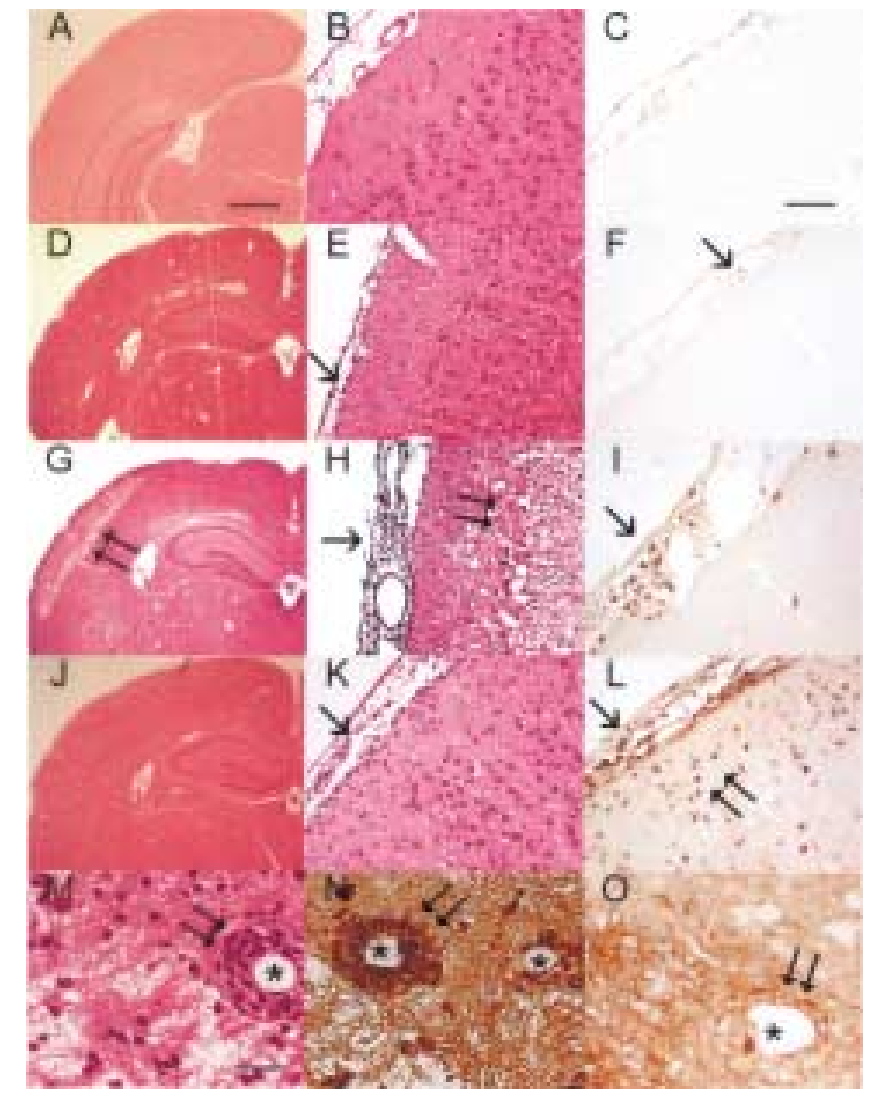

Fig. 4. Representative photomicrographs demonstrating brain histology and immunohistochemical staining for ED 1-positive monocytes. (A-C) Brain of a rat treated with BCG (i.v.) + saline (i.c.v.). (D-F) Brain of a rat treated with saline (i.v.) + LPS (300 mg/rat i.c.v.). Arrows point to occasional meningeal inflammatory cells. (G-I) Rat treated with BCG (i.v.) + LPS (300 mg/rat i.c.v.). Double arrows in panels G and $\mathrm{H}$ mark the infarcted area, single arrows inflammatory cells in meninges (H and I). (J-L) BCG-pretreated rat that was treated with TNFbp before LPS (300 mg/rat i.c.v.). Single arrows mark inflammatory cells in meninges, double arrows in $\mathrm{L}$ denote ED 1-positive parenchymal cells. $M$ depicts perivascular infiltrates (arrows) in a BCG + LPS-treated rat (lumen of the vessel marked with a star). These infiltrates were identified as monocytes with immunostaining with EDl-antibodies (arows in N). Staining of an adjacent section with anti-ICAM-1 antibodies $\mathbf{b}$ demonstrated positive cells in the vascular wall (arrows). Scale bar $=500 \mu \mathrm{m}$ in A, D, G and J, in B, C, E, F, $\mathrm{H}, \mathrm{I}, \mathrm{K}$, and L scale bar $=50 \mu \mathrm{m}$, in $\mathbf{M}-\mathrm{O}$ scale bar $=20 \mu \mathrm{m}$.

staining was more intense in animals in clinical grades 1-2, particularly in the group that received anticytokine treatment (Figure 6F). This staining was completely abolished with preincubation of the antibody with its control peptide (Figure 6G).

Weak cytoplasmic immunoreactivity of IL-6 was seen in some cortical neurons, particularly in animals that survived the LPS challenge (Figure 7A). Intense staining of IL-6 was present in invading inflammatory cells and vascular endothelium of paralyzed and moribund rats, particularly in the cortical areas and white matter areas most severely affected after LPS administration (Figure 7C). Incubation of the anti-

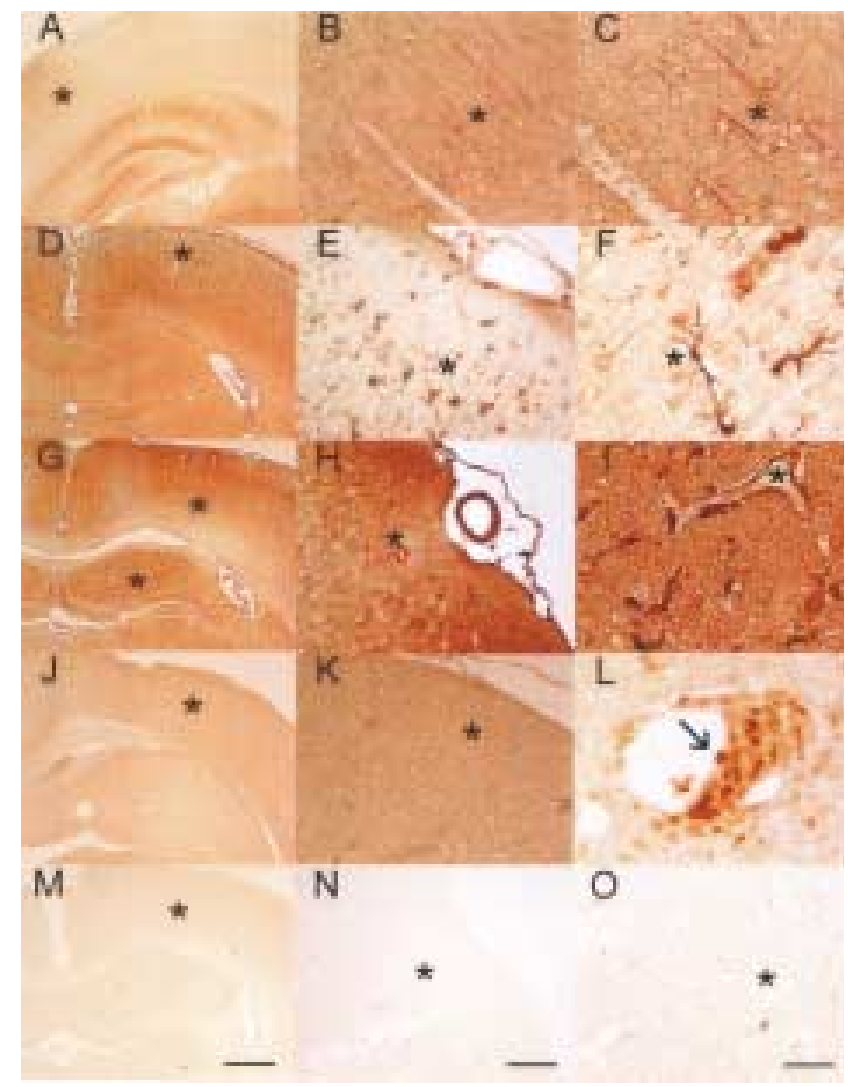

Fig. 5. Expression of IL-1 $\beta$-immunoreactivity in the brain. (A-C) Immunostaining of a brain section from an BCG-primed animal after saline (10 $\mu$ l i.c.v.) treatment, neuronal staining (star) is visible in all panels. (D-F) IL- $1 \beta$-immunoreactivity after LPS treatment in saline-treated rats. Note in D that the microglial staining was restricted to cortex and subcortical white matter (star) but did not involve hippocampus. Panels E and F: higher magnifications of D (star) demostrating both microglial and light neuronal staining. (G-I) IL- $\beta$-immunoreactivity after LPS administration in a BCG-primed rat. Note in panel G strong microglial staining pattern throughout the brain parenchyma (stars). ( $\mathbf{H}$ and $\mathbf{I})$ Higher magnifications of $\mathbf{G}$ demonstrating strong microglial and vascular staining (star). ( $\mathrm{J}$ and $\mathrm{K}$ ) Preadsorption of the signal in G to $\mathbf{H}$. Panel L: IL-1 $\beta$ immunopositive cells (arrow) in perivascular infiltrates of a BCG + LPS-treated rat (adjacent section to that of panels $\mathbf{M}$ through $\mathbf{O}$ in Figure 4). (M-O) Almost complete lack of IL- $1 \beta$ staining in a TNFbp-treated rat's brain. In A, D, G, J, and $\mathbf{M}$ scale bar $=500 \mu \mathrm{m}$, in B, E, H, K, and $\mathbf{N}$ scale bar $=50 \mu \mathrm{m}$, in $\mathbf{C}, \mathbf{F}, \mathbf{I}, \mathbf{L}$, and $\mathbf{O}$ scale bar $=20 \mu \mathrm{m}$.

body with recombinant rat IL-6 abolished the staining in neurons (Figure 7B), blood vessels, and round inflammatory cells (Figure 7D).

\section{Discussion}

The present study examined the role of chronic monocyte/macrophage activation in the development of local thrombosis and hemorrhage using the modified Shwartzman paradigm in rats. Chronic activation of monocytes by BCG injection increased the incidence of paralysis and death, and it produced thrombotic and hemorrhagic infarcts in rats after 
Table 1. Quantitation of IL-1 $\beta$ immunoreactivity in the cortex and hippocampus.

Number of IL-1 $\beta$ Immunoreactive Cells $/ 1 \mathrm{~mm}^{2}$

\begin{tabular}{lcccc}
\hline Group & Cortex & Hippocampus & N & Cell Type Expressing IL-1 $\boldsymbol{\beta}$ \\
\hline Saline i.v. + saline i.c.v. & $58 \pm 23$ & $36 \pm 10$ & 3 & Neurons \\
Saline i.v. + LPS i.c.v. & $107 \pm 30$ & $5 \pm 1$ & 3 & Microglia, monocytes $>$ neurons \\
BCG i.v. + LPS i.c.v. & $124 \pm 19^{\mathrm{x}}$ & $112 \pm 28^{* \mathrm{x}}$ & 4 & Microglia, monocytes $>$ neurons \\
BCG i.v. + ILlra + LPS i.c.v. & $42 \pm 39$ & $32 \pm 29$ & 4 & Microglia, monocytes $>$ neurons \\
BCG i.v. + TNFbp + LPS i.c.v. & $7 \pm 5$ & $2 \pm 1$ & 5 & Microglia, monocytes $>$ neurons
\end{tabular}

Asterisks denote statistical significance in glial IL- $1 \beta$-expression between BCG + LPS and the saline + LPS group $\left({ }^{*} p<0.05\right)$, crosses depict statistical significance between BCG + LPS- and TNF-bp-treated BCG + LPS group $\left({ }^{\mathrm{x}} p<0.01\right)$. A single bolus of saline (10 $\left.\mu \mathrm{l}\right)$ or LPS (100 $\mu \mathrm{g} / \mathrm{rat})$ was administered i.c.v. 2 weeks after the i.v. treatment with BCG or saline. The anticytokine treatments, both at a dose of $100 \mu \mathrm{g} / 10 \mu \mathrm{l}$, were administered i.c.v. $30 \mathrm{~min}$ before and $30 \mathrm{~min}$ after the injection of LPS (100 $\mu \mathrm{g} / \mathrm{rat}$, i.c.v.).

a single i.c.v. injection of LPS. These changes were associated with increased cytokine expression, most profoundly an intense expression of IL-1 $\beta$ immunoreactivity in invading inflammatory cells and microglia. Anticytokine treatments with recombinant soluble TNF receptor antagonist completely blocked, and treatment with recombinant IL-1 receptor antagonist reduced the neurological damage and pathologic lesions in this model. Moreover, the protective effect of anticytokine treatment was correlated with the disappearence of the IL- $1 \beta$ immunoreactivity in microglia and invading inflammatory cells. These results lend support to our hypothesis that monocyte/macrophage activation leads to increased adhesion and transendothelial migration of monocytes. Exaggerated release of IL-1 and TNF- $\alpha$ from these monocyte/macrophages located around local blood vessels mediates preparation of those vessels for focal thrombosis and/or hemorrhage in a paradigm resembling the local Shwartzman reaction $(1,10,11)$.

In our previous study, several established risk factors for stroke (hypertension, old age, diabetes, genetic stroke-proneness) increased the incidence of ischemia and hemorrhage in brainstem vasculature of rats in response to a "provocative" dose of lipopolysaccharide (LPS) (1). We proposed that the stroke risk factor effects may be caused by an increased interaction between cerebrovascular endothelium and circulating monocytes $(1,2,9)$; many studies have provided indirect evidence that support this working hypothesis. Activation of endothelium and monocytes and an increased adherence of monocytes to endothelium are induced by hypertension (3-7). Accordingly, an increased local accumulation of monocyte/macrophages has been found in the brains of aged or stroke-prone and hypertensive rats (8). Cells of these rats produced more $\mathrm{TNF} \alpha$ in response to stimulation (with LPS) than cells from normotensive control animals $(2,6,9)$. These events may contribute to the increased stroke risk in hypertensive and aged animals because the subendothelial clusters of monocyte/macrophages could transform the overlying endothelial cells from an actively anticoagulant state to a prothrombotic state via increased release of TNF- $\alpha$ and IL-1. The present study provides, for the first time, direct evidence that perivascular accumulation of activated monocytes and the production of TNF- $\alpha$ and IL-1 are crucial to the development of neurological deficits and cerebral infarcts after LPS stimulation.

Activation of circulating monocytes was evident after BCG injection in all animals in our study, as demonstrated by the spontaneous superoxide production and adhesion receptor expression. Moreover, monocytes from BCG-primed rats were more adherent to cytokine-stimulated cerebromicrovascular endothelial cell cultures than monocytes from saline-treated rats, and this adhesive interaction was blocked by treatment of the endothelial cells with anti-ICAM-1 antibodies. Reports show that BCG treatment increases urinary output of soluble ICAM1 and secretion of IL- 1 and TNF- $\alpha$ (19). Stimulation of TNF- $\alpha$ release in human monocytes by BCG can be blocked by anti-TNF antibodies (20). IL-1ra partially antagonized LPS-induced cytokine release in monocytes from BCG-treated individuals (21). TNF- $\alpha$ gene expression and blood levels of TNF- $\alpha$ are also upregulated by BCG treatment in mice $(22,23)$. In fact, serum levels of TNF- $\alpha$ after an intraperitoneal injection of LPS were over 200 times higher in mice that had been inoculated with BCG 2 weeks before than in uninfected mice (18). Activated macrophages in granulomas of BCG-treated mice expressed TNF mRNA and produced large amounts of TNF- $\alpha$ (16). TNF released from the granulomas seemed to enhance its own production and favor further macrophage accumulation as treatment with anti-TNF antibodies 


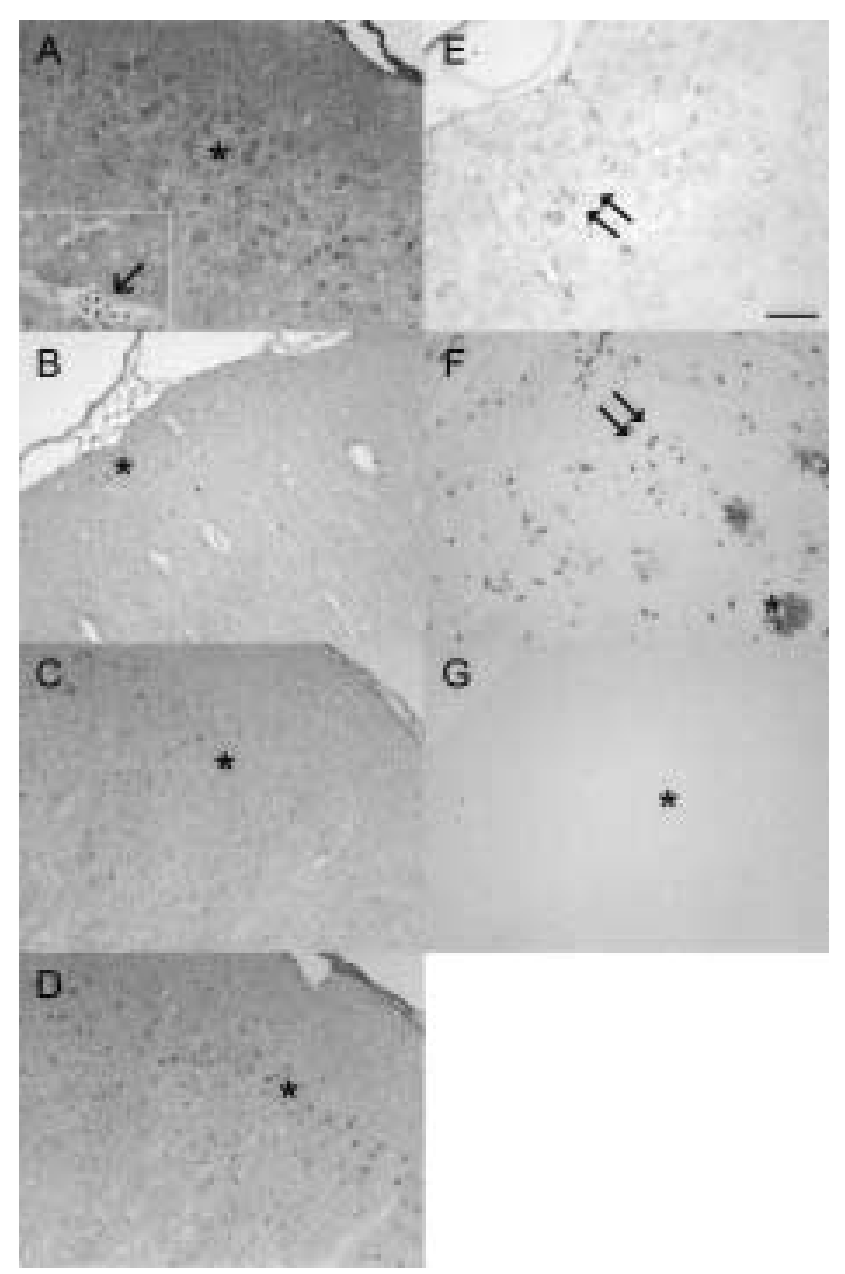

Fig. 6. Expression of TNF $\alpha$ (A-D) and TNFR I (E-G) immunoreactivity in the brain. (A) TNF $\alpha$ immunoreactivity after LPS administration in a BCG-primed rat. Note staining of neurons, small round cells on the cortex (star), in inset staining of monocytes in an intraparenchymal blood vessel (arrow). C preadsoption of the signal in A, with incubation of the antibody with a 50x excess concentration of recombinant rat TNF $\alpha$. D depicts reappearance of the staining when the recombinant rat $\mathrm{TNF} \alpha$ was reacted withTNFbp prior to the antibody preabsorption. (B) TNF $\alpha$ immunoreactivity (star) in the brain of a rat treated with TNFbp. (E) TNFRI immunoreactivity in cortex of a BCG + LPS-treated rat (arrows). (F) TNFR l staining of infiltrating cells (arrows) and granular perivascular staining (star) in a rat treated with TNFbp before LPS. (G) Preadsorption of the signal in F with 100x excess of the control peptide. In all, scale bar $=50 \mu \mathrm{m}$.

prevented the synthesis of TNF and the formation and persistence of macrophage granulomas in response to BCG injections (16). It is likely that a similar process of autoamplification was provoked in our study by in vivo BCG treatment of the rats and that this led to an augmented release of IL-1 and TNF- $\alpha$ from brain intraparenchymal and perivascular cells in response to administration of LPS and to cerebral infarcts that could be prevented by antagonists of TNF- $\alpha$ and IL- 1 actions.

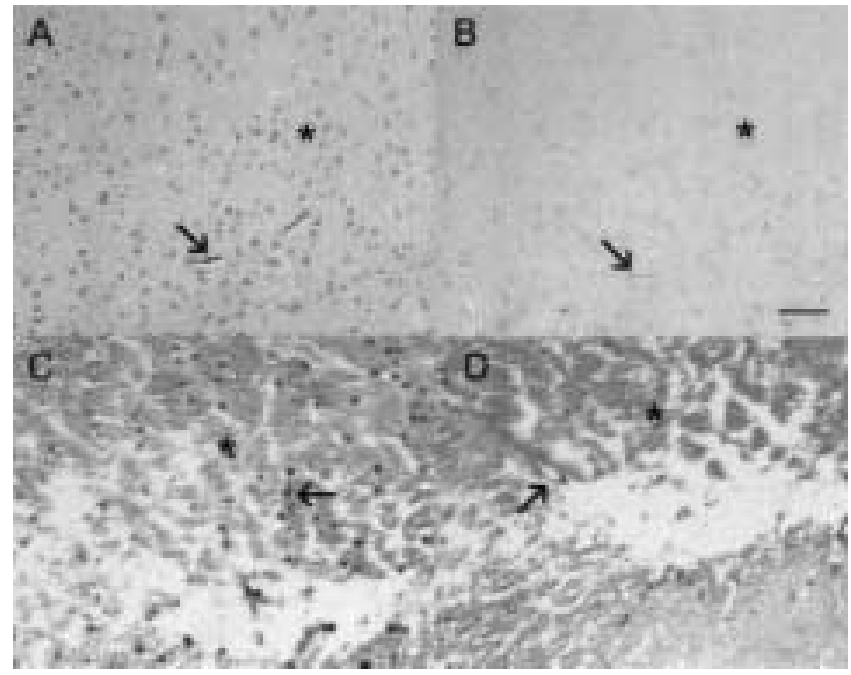

Fig. 7. Expression of IL-6-immunoreactivity in the brain. (A) IL-6 immunoreactivity in cortical neurons (stars) and occasional capillaries (arrow) of the cortex in a surviving rat. (C) IL-6 immunoreactivity in infiltrating cells (star) and endothelium (arrow) after LPS administration in a moribund animal. B demonstrates preadsortion of the signals in A, D preadsoption of signal in $\mathbf{C}$ with $50 x$ excess of recombinant rat IL-6. Scale bar $=50 \mu \mathrm{m}$ in panels.

Cytokine immunoreactivity was found in multiple cell types in control rat brains. Weak-to-moderate expression of TNF- $\alpha$ and IL- $1 \beta$ immunoreactivity was seen in a few cortical neurons, neuronal processes, astrocytes and pericytes of all rats. BCGpriming intensified TNF-staining in invading inflammatory cells, pericytes and white matter microglia. Staining for TNFR 1 in fibers and processes around the cerebral ventricles and brain surface as well as around blood vessels was observed in most animals. There was no clear correlation between severity of disease and that of TNFR I expression. Weak immunoreactivity of IL-6 was seen in cortical neurons and some hippocampal neurons and in microvascular endothelium as well as in meningeal leukocytes in most animals. In BCG-primed rats, an increase of IL- 6 staining of endothelium and invading leukocytes was seen after LPS adminstration. The priming also induced strong IL- $1 \beta$-staining in invading inflammatory cells and microglia most intensively in the cortex, hippocampus and corpus callosum. The histological damage and survival were clearly correlated with the appearance of microglial IL- $1 \beta$-immunoreactivity so that this staining was most intense in animals that suffered brain infarcts and died within $24 \mathrm{hr}$ after LPS administration. Little or no microglial staining with IL- $1 \beta$-antibodies was seen in saline-treated animals or in anticytokine-treated animals that never deteriorated beyond clinical grade 2 (e.g., were not paralyzed). This result implies that microglial IL$1 \beta$ expression may be crucial for the escalation of the disease. Previous studies have demonstrated 
induction of cytokine gene and protein expression after cerebral ischemia (24-28). In agreement with the present data, reports show that IL- $1 \beta$-is expressed in leukocytes, microglia, and astrocytes of the expanding lesion after excitoxic injury (29), trauma (30), or ischemic brain injury (31) and following global cerebral ischemia (26). Focal brain ischemia has also been reported to induce expression of TNF- $\alpha(24,28,32,33)$ and TNFRl(32) immunoreactivity in several cell types of the infarcted brain tissue and its penumbra after middle cerebral artery occlusion.

Recombinant IL-1ra-both at a intracerebroventricular dose of $10 \mu \mathrm{g} 30 \mathrm{~min}$ before and $10 \mathrm{~min}$ after middle cerebral artery occlusion and at a subcutaneous dose of $100 \mathrm{mg} / \mathrm{kg}$ - has produced effective inhibition of IL- 1 activity and has resulted in attenuation of ischemic and excitotoxic damage $(34,35)$. When administered intravenously to rats subjected to focal brain ischemia, TNFbp significantly attenuated the microvessel perfusion impairment observed in vehicle-treated rats. Particularly, the attenuation occurred in the perifocal/penumbral regions of cortex. TNFbp significantly reduced (by 34-38\%) the total volume of ischemic injury (36), and its topical application significantly reduced cortical lesion volume after occlusion of the middle cerebral artery in mice (37). Systemic administration of IL-1ra and TNFbp improved survival of rats after high doses of LPS (38). Our study demonstrates that TNFbp prevents and IL-ra dramatically reduces the development of cerebral infarcts provoked by monocyte activation with BCG and subsequent LPS challenge. The findings support the hypothesis that both TNF$\alpha$ and IL- 1 can mediate initiation of stroke. The fact that IL-Ira did not totally block the effects of LPS in BCG-primed rats in contrast to the TNFbp could result from its relatively short half-life (approximately $6 \frac{1}{2} \mathrm{hr}$ in plasma) $(35,39)$. Interestingly, while ILIra is able to effectively block IL- $1 \beta$ secretion in human monocytes in response to LPS, studies have shown it failed to inhibit the cytokine release in BCG-stimulated monocytes (21). Therefore, it cannot be excluded that the dose of IL-1ra used may have been insufficient to totally block cytokine release in response to the extremely potent stimulus of BCG + LPS.

In conclusion, the results from our present study demonstrate that monocyte activation predisposes brain vessel disruption and occlusion by an exaggerated release of TNF- $\alpha$ and IL-1 from monocyte/macrophages and microglia located around local blood vessels. Our results suggest a crucial role for IL-1 in the generation of brain hemorrhage and ischemia despite that IL-1ra provided only partial protection against LPS-induced strokes. The present data further demonstrated that TNF- $\alpha$ is necessary for infarction to occur in this model. Therefore, TNF$\alpha$ seems to be a vital factor in the circulatory disruption that leads to infarction.

\section{Acknowledgements}

This work has been supported by Public Health Service grant NS-28225 and the Uniformed Services University Protocol R09232. The opinions or assertions contained herein are the private ones of the authors and are not to be construed as official or as necessarily reflecting the views of the Department of Defense or the Uniformed Services University of the Health Sciences. The experiments reported herein were conducted according to the principles set forth in the "Guide for Care and Use of Laboratory Animals," Institute of Laboratory Animal Resources, National Research Council (DHEW Publication No. NIH 85-23, 1985).

\section{References}

1. Hallenbeck JM, Dutka AJ, Kochanek PM, Siren A, Pezeshkpour GH, Feuerstein G. (1988) Stroke risk factors prepare rat brainstem tissues for modified local Shwartzman reaction. Stroke 19: 863-869.

2. Sirén AL, Heldman E, Doron D, et al. (1992) Release of proinflammatory and prothrombotic mediators in the brain and peripheral circulation in spontaneously hypertensive and normotensive Wistar-Kyoto rats. Stroke 23: 1643-1650; discussion 1650-1651.

3. Schmid-Schonbein GW, Seiffge D, DeLano FA, Shen K, Zweifach BW. (1991) Leukocyte counts and activation in spontaneously hypertensive and normotensive. Hypertension 17: 323-330.

4. Duplàa C, Couffinhal T, Labat L, et al. (1993) Monocyte adherence to endothelial cells in patients with atherosclerosis: relationships with risk factors. Eur. J. Clin. Invest. 23: 474-479.

5. McCarron RM, Wang L, Sirén AL, Spatz M, Hallenbeck JM. (1994) Monocyte adhesion to cerebromicrovascular endothelial cells derived from hypertensive and normotensive rats. Am. J. Physiol. 267: H2491-H2497.

6. Liu Y, Liu T, McCarron RM, et al. (1996) Evidence for activation of endothelium and monocytes in hypertensive rats. Am. J. Physiol. 270: H2125-2131.

7. Veniant M, Clozel JP, Kuhn H, Clozel M. (1992) Protective effect of cilazapril on the cerebral circulation. J. Cardiovasc. Pharmacol. 19: S94-S99.

8. Liu Y, Jacobowitz DM, Barone F, et al. (1994) Quantitation of perivascular monocytes and macrophages around cerebral blood vessels of hypertensive and aged rats. J. Cereb. Blood Flow Metab. 14: 348-352.

9. Sirén AL, Liu Y, Feuerstein G, Hallenbeck JM. (1993) Increased release of tumor necrosis factor-alpha into the cerebrospinal fluid and peripheral circulation of aged rats. Stroke 24: 880-886; discussion 887-888.

10. Movat HZ, Burrowes CE, Cybulsky MI, Dinarello CA. (1987) Acute inflammation and a Shwartzman-like reaction induced by interleukin-1 and tumor necrosis factor. Synergistic action of the cytokines in the induction of inflammation and microvascular injury. Am. J. Pathol. 129: 463-476.

11. Shwartzman G. (1928) Studies on Bacillus typhosus toxic substances -I. Phenomenon of local skin reactivity to B. typhosus culture filtrate. J. Exp. Med. 48: 247-268.

12. Strassmann G, Springer TA, Haskill SJ, Miraglia CC, Lanier LL, Adams DO. (1985) Antigens associated with the activation of murine mononuclear phagocytes in vivo: differential expression of lymphocyte function-associated antigen in the several stages of development. Cell Immunol. 94: 265-275.

13. Vogel SN, Moore RN, Sipe JD, Rosenstreich DL. (1980) BCGinduced enhancement of endotoxin sensitivity in $\mathrm{C} 3 \mathrm{H} / \mathrm{HeJ}$ mice. I. In vivo studies. J. Immunol. 124: 2004-2009. 
14. Perper RJ, Oronsky AL, Sanda M (1976) The effect of BCG on extravascular mononuclear cell accumulation in vivo. Int. J. Cancer 17: 670-677.

15. Blanden RV, Lefford MJ , Mackaness GB. (1969) The host response to Calmette-Guerin bacillus infection in mice. J. Exp. Med. 129: 1079-1107.

16. Kindler V, Sappino AP, Grau GE, Piguet PF, Vassalli P. (1989) The inducing role of tumor necrosis factor in the development of bactericidal granulomas during BCG infection. Cell 56: 731-740.

17. Izzo AA, North RJ. (1992) Evidence for an alpha/beta T cellindependent mechanism of resistance to mycobacteria. Bacillus-Calmette-Guerin causes progressive infection in severe combined immunodeficient mice, but not in nude mice or in mice depleted of CD4+ and CD8+ T cells. J. Exp. Med. 176: 581-586.

18. Kamijo R, Le J, Shapiro D, et al. (1993) Mice that lack the interferon-gamma receptor have profoundly altered responses to infection with Bacillus Calmette-Guerin and subsequent challenge with lipopolysaccharide. J. Exp. Med. 178: 1435-1440.

19. Jackson AM, Alexandroff AB, Kelly RW, et al. (1995) Changes in urinary cytokines and soluble intercellular adhesion molecule-1 (ICAM-1) in bladder cancer patients after bacillus Calmette- Guerin (BCG) immunotherapy. Clin. Exp. Immunol. 99: 369-375.

20. Fazal N, Lammas DA, Raykundalia C, Bartlett R, Kumararatne DS. (1992) Effect of blocking TNF-alpha on intracellular BCG (Bacillus Calmette Guerin) growth in human monocyte-derived macrophages. FEMS Microbiol. Immunol. 5: 337-345.

21. Conti P, Reale M, Nicolai M, et al. (1994) Bacillus CalmetteGuerin potentiates monocyte responses to lipopolysaccharide-induced tumor necrosis factor and interleukin-1, but not interleukin-6 in bladder cancer patients. Cancer Immunol. Immunother. 38: 365-371.

22. Le Contel C, Beigneux AP, Huang J, Parant MA. (1995) Regulation of lipopolysaccharide-induced tumor necrosis factor production by cyclosporin A in mice primed with muramyl dipeptide. FEMS Immunol. Med. Microbiol. 11: 297-305.

23. Shin JS, Park JH, Kim JD, Lee JM, Kim SJ. (1995) Induction of tumour necrosis factor-alpha (TNF-alpha) mRNA in bladders and spleens of mice after intravesical administration of bacillus Calmette-Guerin. Clin. Exp. Immunol. 100: 26-31.

24. Liu T, Clark RK, McDonnell PC, et al. (1994) Tumor necrosis factor-alpha expression in ischemic neurons. Stroke 25: 1481-1488.

25. Liu T, McDonnell PC, Young PR, et al. (1993) Interleukin-1 beta mRNA expression in ischemic rat cortex. Stroke 24: 1746-1750; discussion 1750-1751.

26. Sairanen TR, Lindsberg PJ, Brenner M, Sirén AL. (1997) Global forebrain ischemia results in differential cellular expression of interleukin-1beta (IL-1beta) and its receptor at mRNA and protein level. J. Cereb. Blood Flow Metab. 17: 1107-1120.
27. Buttini M, Sauter A, Boddeke HW. (1994) Induction of interleukin-1 beta mRNA after focal cerebral ischaemia in the rat. Mol. Brain Res. 23: 126-134.

28. Buttini M, Appel K, Sauter A, Gebicke-Haerter PJ, Boddeke HW. (1996) Expression of tumor necrosis factor alpha after focal cerebral ischaemia in the rat. Neuroscience 71: 1-16.

29. Pearson VL, Rothwell NJ, Toulmond S. (1999) Excitotoxic brain damage in the rat induces interleukin-1beta protein in microglia and astrocytes: correlation with the progression of cell death. Glia 25: 311-323.

30. Knerlich F, Schilling L, Görlach C, Wahl M, Ehrenreich H, Sirén A-L. (1999) Temporal profile of expression and cellular localization of inducible nitric oxide synthase, interleukin-lbeta and interleukin converting enzyme after cryogenic lesion of the rat parietal cortex. Mol. Brain Res. 68: 73-87.

31. Davies CA, Loddick SA, Toulmond S, Stroemer RP, Hunt J, Rothwell NJ. (1999) The progression and topographic distribution of interleukin-lbeta expression after permanent middle cerebral artery occlusion in the rat. J. Cereb. Blood Flow Metab. 19: 87-98.

32. Botchkina GI, Meistrell ME, 3rd, Botchkina IL, Tracey KJ. (1997) Expression of TNF and TNF receptors (p55 and p75) in the rat brain after focal cerebral ischemia. Mol. Med. 3: $765-781$.

33. Nawashiro H, Tasaki K, Ruetzler CA, Hallenbeck JM. (1997) TNF-alpha pretreatment induces protective effects against focal cerebral ischemia in mice. J. Cereb. Blood Flow Metab. 17: 483-490.

34. Relton JK, Rothwell NJ. (1992) Interleukin-1 receptor antagonist inhibits ischaemic and excitotoxic neuronal damage in the rat. Brain Res. Bull. 29: 243-246.

35. Relton JK, Martin D, Thompson RC, Russell DA. (1996) Peripheral administration of Interleukin-1 Receptor antagonist inhibits brain damage after focal cerebral ischemia in the rat. Exp. Neurol. 138: 206-213.

36. Dawson DA, Martin D, Hallenbeck JM. (1996) Inhibition of tumor necrosis factor-alpha reduces focal cerebral ischemic injury in the spontaneously hypertensive rat. Neurosci. Lett. 218: 41-44.

37. Nawashiro H, Martin D, Hallenbeck JM. (1997) Inhibition of tumor necrosis factor and amelioration of brain infarction in mice. J. Cereb. Blood Flow Metab. 17: 229-232.

38. Russell DA, Tucker KK, Chinookoswong N, Thompson RC, Kohno T. (1995) Combined inhibition of interleukin-1 and tumor necrosis factor in rodent endotoxemia: improved survival and organ function. J. Infect. Dis. 171: 1528-1538.

39. Martin D, Chinookoswong N, Miller G. (1994) The interleukin-1 receptor antagonist (rhIL-lra) protects against cerebral infarction in a rat model of hypoxia-ischemia. Exp. Neurol. 130: 362-367. 